University of Nebraska - Lincoln

DigitalCommons@University of Nebraska - Lincoln

2-22-1997

\title{
Vapor-liquid coexistence of quasi-two-dimensional Stockmayer fluids
}

G.T. Gao

University of Nebraska-Lincoln

Xiao Cheng Zeng

University of Nebraska-Lincoln, xzeng1@unl.edu

Wenchuan Wang

Beijing University of Chemical Technology, Beijing 100029, People's Republic of China

Follow this and additional works at: https://digitalcommons.unl.edu/chemzeng

Part of the Chemistry Commons

Gao, G.T.; Zeng, Xiao Cheng; and Wang, Wenchuan, "Vapor-liquid coexistence of quasi-two-dimensional Stockmayer fluids" (1997). Xiao Cheng Zeng Publications. 71.

https://digitalcommons.unl.edu/chemzeng/71

This Article is brought to you for free and open access by the Published Research - Department of Chemistry at DigitalCommons@University of Nebraska - Lincoln. It has been accepted for inclusion in Xiao Cheng Zeng Publications by an authorized administrator of DigitalCommons@University of Nebraska - Lincoln. 


\title{
Vapor-liquid coexistence of quasi-two-dimensional Stockmayer fluids
}

\author{
G. T. Gao a) and X. C. Zeng ${ }^{\text {b) }}$ \\ Chemistry Department and Center for Materials Research and Analysis, University of Nebraska-Lincoln, \\ Lincoln, Nebraska 68588 \\ Wenchuan Wang \\ Department of Chemical Engineering, Beijing University of Chemical Technology, Beijing 100029, \\ People's Republic of China
}

(Received 12 November 1996; accepted 22 November 1996)

\begin{abstract}
A quasi-two-dimensional (2D) Stockmayer model is developed in which the center of mass of the molecule is confined on a plane while the dipole of the molecule can rotate freely in three dimensional space. This model entails essential characteristics of systems such as dipolar molecules physisorbed on a solid surface, or a Langmuir monolayer consisting of short-chain molecules with a dipolar tail. The Gibbs ensemble Monte Carlo technique is employed to determine the vaporliquid equilibria of the model fluids. An Ewald sum for this quasi-2D model is formulated to account for the long-range dipolar interactions. Three systems with different reduced dipole moments were studied. The critical point of each system is determined by fitting the vapor-liquid coexistence data to a $2 \mathrm{D}$ scaling law and the rectilinear law. We find that in general the critical temperature of the system is reduced due to the confinement and is sensitive to the strength of the dipole moment, whereas the critical density is not. The effect of reducing the dispersion part of potential on the vapor-liquid equilibria is also studied. We find the dispersion potential reduction leads to a lower critical temperature and a higher in-plane part of molecular dipole moment; however, because the reduced critical temperature is relatively small compared with that of a 3D system, disappearance of the critical point is not observed in the quasi-2D SM system within practical scope of the simulation.

(C) 1997 American Institute of Physics. [S0021-9606(97)51508-X]
\end{abstract}

\section{INTRODUCTION}

Although strict 2D systems do not exist in the real world, many realistic systems exhibit 2D or quasi-2D characteristics. Examples are physisorption of gases on solid surfaces, ${ }^{1}$ surfactant monolayers adsorbed on an air/water interface, ${ }^{2}$ and thinning by evaporation of completely wetted liquid films on clean solid surfaces. ${ }^{3}$ By use of computer simulations, however, exact 2D systems can be studied. Indeed, in the past two decades, computer simulations have allowed considerable progress toward understanding exact 2D fluid systems; for example, the equation of state, ${ }^{4}$ critical phenomena, ${ }^{5,6}$ and the liquid-vapor interface ${ }^{5,7,8}$ of $2 \mathrm{D}$ Lennard-Jones (LJ) system have been studied. In recent years, there has been growing interest in the determination of vapor-liquid coexistence curve for the $2 \mathrm{D} \mathrm{LJ}$ fluids by use of the newly developed Gibbs ensemble Monte Carlo technique. $^{9-11}$ As 3D systems, different phases of a 2D system can coexist under the equilibrium condition, which is the equality of temperature, pressure, and chemical potential of each phase. ${ }^{12}$

Although physisorbed nonpolar molecules or thin molecular films with nonpolar tails can be modeled by an exact 2D LJ system when their vapor-liquid coexistence is concerned, ${ }^{11}$ physisorbed polar molecules or thin-films with polar tails are intrinsically quasi-2D in nature. On one hand,

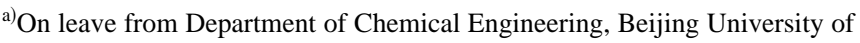
Chemical Technology, Beijing 100029, People's Republic of China.

b) Author to whom correspondence should be addressed.
}

the position of the molecules is confined on a surface; on the other hand, the dipole moments of the molecules may take any direction in $3 \mathrm{D}$.

In this paper we devise a simple model - the quasi-2D Stockmayer (SM) model-which is a confined dipolar LJ system. In this model, the molecules can move only within a plane while the dipoles of the molecules can freely rotate in $3 \mathrm{D}$. Note that this model is essentially an off-lattice 2D Heisenberg model. ${ }^{22}$ Note also that the quasi-2D SM differs from the exact 2D SM (which is also the off-lattice $X-Y$ model) in that for the latter model the dipoles of molecules allow to rotate only in the same plane as the translational movement and the dipole-dipole interaction energy is determined from the solution of the $2 \mathrm{D}$ Laplace equation. ${ }^{13} \mathrm{Al}-$ though several computational studies ${ }^{13}$ have been undertaken for the exact 2D SM systems, the present work is, to our knowledge, the first computer simulation for a quasi-2D SM system.

The focus of this work is the vapor-liquid phase equilibria and the critical point of the quasi-2D SM system. We explore how the molecular polarity affects the vapor-liquid phase equilibria and the critical point. We also attempt to look into whether the vapor-liquid transition exists when the dispersion part of the SM potential is reduced. In this work we employ the Gibbs ensemble Monte Carlo molecular simulation technique to derive the vapor-liquid coexistence curves. The paper is organized as follows. In Sec. II, we describe the quasi-2D SM model and present details of the simulation. Section III includes discussion of the simulation results for three systems having different reduced dipole 
moments and the effect of reducing the dispersion part of the potential on the phase equilibria. The conclusion is given in Sec. IV. In the appendices, we show a derivation of the Ewald summation formula for the quasi-2D SM system, which is used to treat the long-range dipole-dipole interactions in the computer simulations.

\section{MODEL AND SIMULATION METHOD}

The SM potential model describes the intermolecular interaction by adding a dipole-dipole interaction term to the LJ potential, i.e.,

$$
U_{i j}\left(\mathbf{r}_{\mathbf{i j}}, \boldsymbol{\mu}_{\mathbf{i}}, \boldsymbol{\mu}_{\mathbf{j}}\right)=4 \epsilon\left[\left(\frac{\sigma}{r_{i j}}\right)^{12}-\left(\frac{\sigma}{r_{i j}}\right)^{6}\right]+U_{D}\left(\mathbf{r}_{\mathrm{rij}}, \boldsymbol{\mu}_{\mathbf{i}}, \boldsymbol{\mu}_{\mathbf{j}}\right) .
$$

The first part of Eq. (1) is the LJ potential energy where $r_{i j}$ is the distance between particle $i$ and $j$, and $\epsilon$ and $\sigma$ are the energy and size parameters. $U_{D}$ is the dipole-dipole interaction energy which can be expressed as

$$
U_{D}\left(\mathbf{r}_{\mathbf{i j}}, \boldsymbol{\mu}_{\mathbf{i}}, \boldsymbol{\mu}_{\mathbf{j}}\right)=\frac{1}{r_{i j}^{3}}\left[\boldsymbol{\mu}_{\mathbf{i}} \cdot \boldsymbol{\mu}_{\mathbf{j}}-\frac{3}{r_{i j}^{2}}\left(\boldsymbol{\mu}_{\mathbf{i}} \cdot \mathbf{r}_{\mathbf{i j}}\right)\left(\boldsymbol{\mu}_{\mathbf{j}} \cdot \mathbf{r}_{\mathbf{i j}}\right)\right],
$$

where $\mu$ is the molecular dipole moment. In a quasi-2D SM system, the positions of the molecules are confined in a 2D plane while the dipoles of the molecules can rotate freely in a 3D space.

Computer simulations for a system involving dipoles are more time consuming because of the long-range nature of dipole-dipole interactions. The standard cutoff approximation cannot be adopted with the common simulation size. Instead, special techniques such as the Ewald sum or reaction field are needed to handle the long-range interactions. Several general treatments ${ }^{14-17}$ in deriving the Ewald sum formula were developed and used in computer simulations of 3D SM systems. Here we adapt the Nijbore and de Wette approach ${ }^{17}$ to derive an Ewald sum formula for the quasi-2D SM system. In doing so, we divide the molecular dipole moment into two parts; (1) $\mu_{s}$, the in-plane part, and (2) $\mu_{z}$, the part which is normal to the plane. Since for the quasi-2D SM system $\mathbf{r}_{\mathbf{i j}}$ is within the $2 \mathrm{D}$ plane, $U_{D}$ in Eq. (2) can be written as

$$
U_{D}=\frac{1}{r_{i j}^{3}}\left[\boldsymbol{\mu}_{\mathrm{is}} \cdot \boldsymbol{\mu}_{\mathrm{js}}-\frac{3}{r_{i j}^{2}}\left(\boldsymbol{\mu}_{\mathrm{is}} \cdot \mathbf{r}_{\mathrm{ij}}\right)\left(\boldsymbol{\mu}_{\mathrm{js}} \cdot \mathbf{r}_{\mathrm{ij}}\right)\right]+\frac{\mu_{i z} \mu_{j z}}{r_{i j}^{3}} .
$$

With such a division, $U_{D}$ can be separated into two parts, one related only to $\mu_{s}$, and the other related only to $\mu_{z}$. Thus the Ewald sum for each part can be derived separately. The details of the derivation are given in Appendix A.

To obtain the phase equilibria for the quasi-2D SM system, the NVT Gibbs ensemble Monte Carlo (GEMC) technique is employed. This technique was developed by Panagiotopoulos in 1987. ${ }^{18}$ Details of this method can be found in the original papers of Panagiotopoulos ${ }^{18}$ Panagiotopoulos et al. ${ }^{19}$ and in the review article of Panagiotopoulos. ${ }^{20}$ In a GEMC simulation, two homogenous fluid phases at thermodynamic equilibrium are simulated simultaneously in two separated boxes. Three types of Monte Carlo moves are in-
TABLE I. Definitions of reduced quantities. ${ }^{\text {a }}$

\begin{tabular}{lcc}
\hline \hline Reduced dipole moment & $\mu^{*}$ & $\sqrt{\mu^{2} / \epsilon \sigma^{3}}$ \\
Reduced pressure & $P^{*}$ & $P \frac{\sigma^{2}}{\epsilon}$ \\
Reduced temperature & $T^{*}$ & $\frac{k_{B} T}{\epsilon}$ \\
Reduced density & $\rho^{*}$ & $\frac{N}{S} \sigma^{2}$ \\
Reduced potential energy per molecule & $E^{*}$ & $\frac{E}{N \epsilon}$ \\
Reduced chemical potential & & $\frac{c}{\epsilon}$ \\
\end{tabular}

${ }^{\text {a } P=2 D}$ pressure; $N=$ number of molecules; $T=$ temperature; $k_{B}=$ Boltzmann constant; $S=2$ D volume; $E=$ total potential energy; $c=$ chemical potential.

volved; (1) particle displacement for internal equilibrium; (2) particle interchange for chemical equilibrium; and (3) volume rearrangement for mechanical equilibrium. In our simulations, we used 512 particles in every simulation run. With this particle number, no appreciable finite-size effects were found. Simulations are conducted in cycles; each cycle contains 512 particle displacements, 200 to 500 particle interchanges, and one volume rearrangement. A complete simulation typically involves 20000 cycles. The first 10000 cycles are used for equilibration and the second 10000 cycles are used to accumulate data. Normally, the final 10000 cycles are divided into 10 blocks; the standard deviations are calculated from block averages.

The LJ part of molecular interaction potential is truncated at the distance equivalent to half box length; longrange correction is included by assuming the radial distribution function, $g(r)=1$, for distances beyond the cutoff radius. When the Ewald sum is used, the "tinfoil"' boundary condition ${ }^{15}$ is adopted, and a careful pretest shows that the optimal value of parameter $\kappa$ (which governs the convergence of the Ewald sum; see Appendix A) is about 5.0, and that a total of 53 reciprocal lattice vectors is sufficient to give convergent results.

The chemical potentials are also calculated from the equation proposed by Smit and Frenkel. ${ }^{21}$ Unlike the LJ system, caution must be taken when the energy change for a test particle insertion is calculated. This is because, when the Ewald sum is used to handle the long-range interactions, the energy change for inserting the test particle into the system contains the interaction between the inserted particle with all its images. The test particle-image interactions must be $e x$ cluded from the test particle-system interactions.

\section{RESULTS AND DISCUSSION}

In the following, all quantities are shown in reduced units; the definitions for the reduced units are given in Table I. In order to examine the dipolar effects on the vapor-liquid coexistence for the quasi-2D SM system, we have investigated three systems with the reduced dipole moment $\mu^{*}=1$, $\sqrt{2}$, and $\sqrt{ } 3$, respectively. Using the GEMC simulation tech- 
TABLE II. Simulation results for quasi 2-D SM fluid. See Table I for the symbols. The values in the parentheses indicate the uncertainty in the last $\operatorname{digit}(\mathrm{s})$.

\begin{tabular}{|c|c|c|c|c|c|c|c|c|}
\hline \multirow[b]{2}{*}{$T^{*}$} & \multicolumn{4}{|c|}{ Vapor phase } & \multicolumn{4}{|c|}{ Liquid phase } \\
\hline & $\rho_{g}^{*}$ & $P_{g}^{*}$ & $-E_{g}^{*}$ & $-c_{g}^{*}$ & $\rho_{l}^{*}$ & $P_{l}^{*}$ & $-E_{l}^{*}$ & $-c_{l}^{*}$ \\
\hline \multicolumn{9}{|c|}{$\mu^{*}=1.0$} \\
\hline 0.54 & $0.0227(9)$ & $0.0101(3)$ & $0.322(34)$ & $2.25(2)$ & $0.736(8)$ & $0.00(2)$ & $3.21(3)$ & $2.24(14)$ \\
\hline 0.55 & $0.035(4)$ & $0.0138(13)$ & $0.54(11)$ & $2.15(3)$ & $0.740(6)$ & $0.02(2)$ & $3.21(3)$ & $2.20(16)$ \\
\hline 0.56 & $0.041(3)$ & $0.0153(11)$ & $0.64(6)$ & $2.13(3)$ & $0.723(7)$ & $0.015(14)$ & $3.12(3)$ & $2.24(23)$ \\
\hline 0.57 & $0.046(5)$ & $0.0169(11)$ & $0.72(15)$ & $2.11(3)$ & $0.706(11)$ & $0.010(13)$ & $3.05(4)$ & $2.22(13)$ \\
\hline 0.58 & $0.057(3)$ & $0.0205(12)$ & $0.74(7)$ & $2.08(2)$ & $0.701(13)$ & $0.023(15)$ & $3.02(4)$ & $2.18(16)$ \\
\hline 0.59 & $0.116(12)$ & $0.0301(19)$ & $1.13(13)$ & $2.04(4)$ & $0.642(30)$ & $0.027(9)$ & $2.70(8)$ & $2.34(26)$ \\
\hline \multicolumn{9}{|c|}{$\mu^{*}=\sqrt{ } 2$} \\
\hline 0.72 & $0.040(4)$ & $0.0172(11)$ & $1.20(21)$ & $2.86(14)$ & $0.742(10)$ & $0.012(21)$ & $4.68(6)$ & $2.93(36)$ \\
\hline 0.73 & $0.038(3)$ & $0.0176(18)$ & $1.10(15)$ & $2.85(4)$ & $0.717(21)$ & $0.028(17)$ & $4.56(7)$ & 2.95(47) \\
\hline 0.74 & $0.071(4)$ & $0.021(3)$ & $2.10(15)$ & $2.81(33)$ & $0.714(8)$ & $0.026(28)$ & $4.50(3)$ & $2.95(25)$ \\
\hline 0.75 & $0.069(10)$ & $0.024(3)$ & $1.79(43)$ & $2.77(9)$ & $0.685(22)$ & $0.020(20)$ & $4.35(8)$ & $2.82(28)$ \\
\hline 0.76 & $0.089(15)$ & $0.027(4)$ & $1.88(30)$ & 2.74(9) & $0.685(9)$ & $0.034(21)$ & $4.32(5)$ & $2.87(23)$ \\
\hline 0.77 & $0.207(26)$ & $0.031(12)$ & $3.13(23)$ & $2.73(27)$ & $0.44(7)$ & $0.027(14)$ & $3.76(14)$ & $3.02(21)$ \\
\hline \multicolumn{9}{|c|}{$\mu^{*}=\sqrt{ } 3$} \\
\hline 0.88 & $0.053(3)$ & $0.016(5)$ & $3.40(26)$ & $3.57(21)$ & $0.755(14)$ & $0.022(22)$ & $6.46(8)$ & $3.9(6)$ \\
\hline 0.89 & $0.064(5)$ & $0.020(5)$ & $3.77(17)$ & $3.8(1.1)$ & $0.741(13)$ & $0.014(25)$ & $6.38(6)$ & $3.64(30)$ \\
\hline 0.90 & $0.073(4)$ & $0.019(4)$ & $3.66(14)$ & $3.39(20)$ & $0.733(16)$ & $0.012(30)$ & $6.32(8)$ & $4.0(4)$ \\
\hline 0.91 & $0.075(6)$ & $0.017(5)$ & $4.17(12)$ & $3.6(5)$ & $0.721(24)$ & $0.026(30)$ & $6.26(11)$ & $3.94(35)$ \\
\hline 0.02 & $0.093(5)$ & $0.022(6)$ & 4.11(14) & $3.9(6)$ & $0.687(39)$ & $0.024(33)$ & $6.15(17)$ & $4.20(31)$ \\
\hline 0.93 & $0.158(8)$ & $0.021(17)$ & 4.63(18) & $3.4(5)$ & $0.504(28)$ & $0.023(23)$ & $5.73(8)$ & $3.9(5)$ \\
\hline
\end{tabular}

nique, the coexistence curves are determined for each system. Simulation results, together with their standard deviations, are given in Table II. The vapor-liquid coexistence curves for the three systems are shown in Fig. 1.

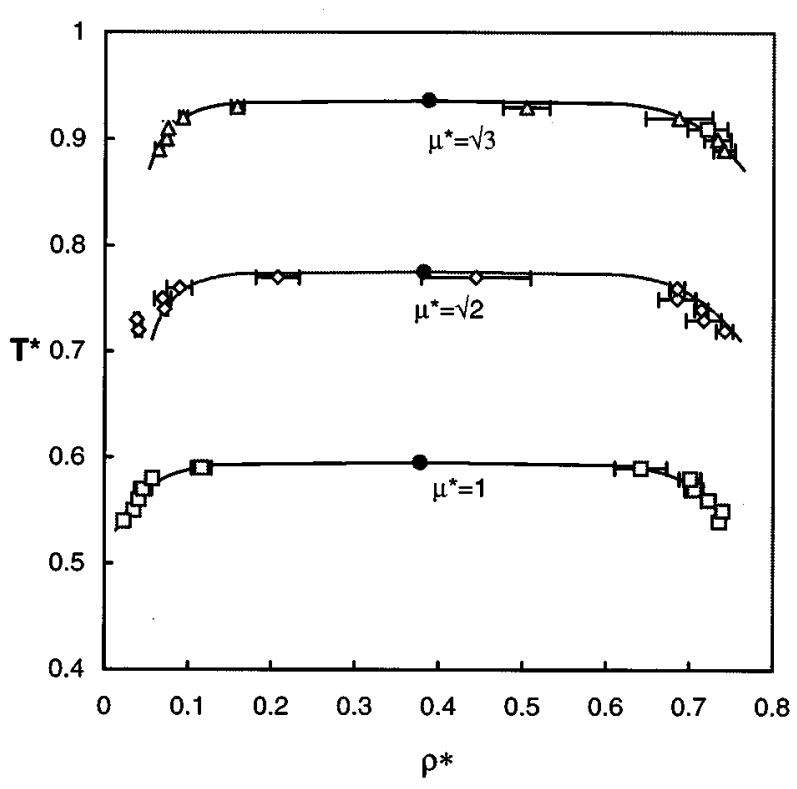

FIG. 1. Phase diagrams of the quasi-2D SM fluids at three reduced dipole moments. The square, diamond, and triangle dots are the GEMC simulation results. The solid circles are the estimates of the critical points based on the simulation results. The lines are the fits to a $2 \mathrm{D}$ scaling law and the rectilinear law.
Although the critical point cannot be determined directly from the simulation, one can estimate the critical point by fitting the vapor-liquid coexistence data to the scaling law,

$$
\rho_{l}^{*}-\rho_{v}^{*}=A\left(T_{c}^{*}-T^{*}\right)^{\beta}
$$

and the rectilinear law,

$$
\frac{\rho_{l}^{*}+\rho_{v}^{*}}{2}=\rho_{c}^{*}+B\left(T_{c}^{*}-T^{*}\right),
$$

where $T_{c}^{*}$ is the reduced critical temperature, $\rho_{c}^{*}$ is the reduced critical density, $A$ and $B$ are two associated coefficients, and $\beta$ is the critical exponent. For $2 \mathrm{D}$ systems, it is well known that the rigorous result of $\beta$ is 0.125 , and the result of $\beta$ under mean-field assumption is $0.5{ }^{22}$ However, Recht and Panagiotopoulos ${ }^{23}$ have pointed out that, as the critical point is approached, a crossover from rigorous to mean-field scaling exponent occurs for 2D systems. This indicates that data very close to the critical point should be used cautiously in the fitting. We have examined our simulation data and found that nearly all data can be fitted by using the rigorous scaling exponent $(\beta=0.125)$, except the data for $\mu^{*}=\sqrt{ } 2$ at $T^{*}=0.77$ and for $\mu^{*}=\sqrt{ } 3$ at $T^{*}=0.93$, which seem to be too close to the critical point and are thus excluded from the fitting. Using Eqs. (4) and (5), we estimate the critical point for the three systems. Results of the reduced critical temperatures and the densities are shown in Table III.

From Table III one can see that the critical temperature is sensitive to the strength of the dipole moment, i.e., a larger reduced dipole moment leads to a higher critical temperature. This is because the effective dipole-dipole interaction is at- 
TABLE III. Reduced critical temperatures and densities for quasi-2D SM fluids with three reduced dipole moments.

\begin{tabular}{ccc}
\hline \hline$\mu^{*}$ & $T_{c}^{*}$ & $\rho_{c}^{*}$ \\
\hline 1.0 & 0.595 & $0.378 \pm 0.007$ \\
$\sqrt{2}$ & 0.775 & $0.382 \pm 0.011$ \\
$\sqrt{ } 3$ & 0.936 & $0.388 \pm 0.013$ \\
\hline \hline
\end{tabular}

tractive in nature. The larger the reduced molecular dipole moments, the stronger the attractive force, and thus the more difficult to separate two molecules in liquid. On the other hand, one can also find the critical density almost does not change as the reduced dipole moments varies. These two trends have also been found in the 3D SM system. ${ }^{24}$

It is instructive to compare the reduced critical temperature of the $2 \mathrm{D}$ systems with that of 3D systems. Comparing our results with those of van Leeuwen, we find that the ratios of the quasi-2D to the 3D critical temperature are $0.42,0.48$, and 0.51 , respectively for $\mu^{*}=1, \sqrt{ } 2$, and $\sqrt{ } 3$. An experimental study for the 2D adsorbed systems shows that the ratio always seems close to 0.4 (between 0.36 and 0.56 ). ${ }^{25}$ Note also that this ratio is 0.37 for the Ising model (from computer simulation) and 0.5 using the mean-field theory.

Although the dipolar interaction is effectively attractive, it is very different from the attractive dispersion interaction given by the power- 6 term in the LJ model; the former is anisotropic, whereas the latter is isotropic. It was shown ${ }^{26}$ that the dipolar interaction tends to aggregate the molecules into anisotropic chainlike structures while the LJ interaction favors the isotropic dropletlike structures. Recently, van Leeuwen and Smit found ${ }^{27}$ that a minimum amount of isotropic attractive energy is necessary to stabilize the vaporliquid coexistence for a 3D system interacting with the potential,

$$
\begin{aligned}
U_{i j}\left(\mathbf{r}_{i j}, \boldsymbol{\mu}_{i}, \boldsymbol{\mu}_{j}\right)= & 4 \epsilon\left[\left(\frac{\sigma}{r_{i j}}\right)^{12}-\lambda\left(\frac{\sigma}{r_{i j}}\right)^{6}\right] \\
& +U_{D}\left(\mathbf{r}_{i j}, \boldsymbol{\mu}_{i}, \boldsymbol{\mu}_{j}\right),
\end{aligned}
$$

where the LJ dispersion term is reduced by a factor $\lambda(0<\lambda$ $<1$ ). Using GEMC simulations, they found that as the $\lambda$ is reduced, there exists a threshold for $\lambda$ below which the vapor-liquid coexistence cannot be found; instead, a head to tail chainlike structure forms. Later simulation studies also support this finding. ${ }^{27-29} \mathrm{Can}$ similar behavior happen for the quasi-2D system if the dispersion energy is also reduced? To address this question, we have performed GEMC simulations with a potential similar to Eq. (6). In the simulations, $\mu^{* 2}$ is set to be 1. Vapor-liquid coexistence curves for $\lambda=0.95,0.7$ and 0.5 are obtained and shown in Fig. 2. For comparison, the curve for $\lambda=1$ is also shown in this figure.

As found in the 3D system by van Leeuwen and Smit, when $\lambda$ decreases, the critical temperature of the quasi-2D system also decreases due to the weakening of the attractive dispersion force. However, when $\lambda$ is reduced to 0.5 , we find the successful exchange rate in GEMC simulations becomes lower than $0.1 \%$ at the reduced temperature $T^{*}=0.2$. This

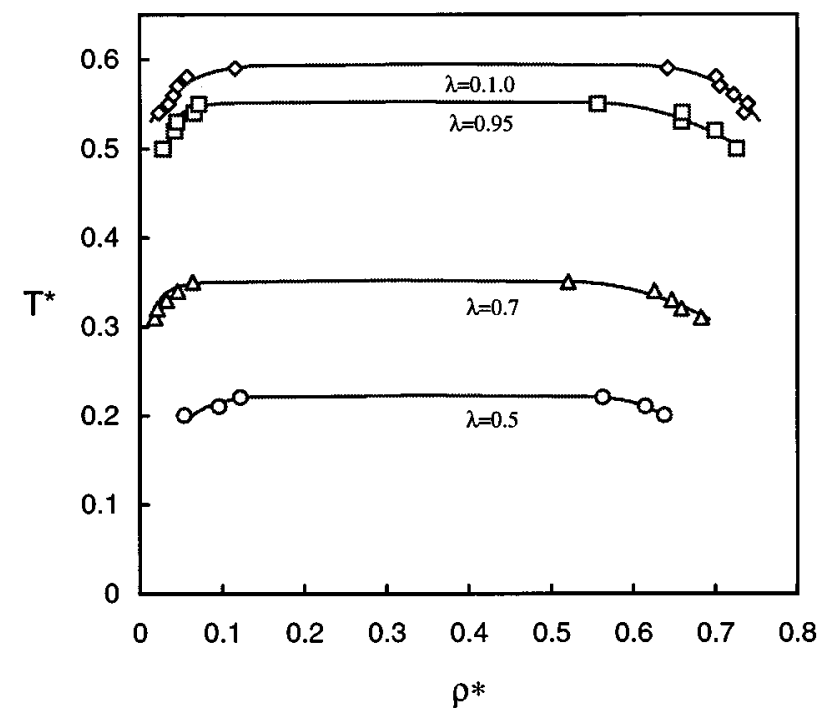

FIG. 2. Phase diagrams of the quasi-2D SM fluids when the dispersion term of the potential function is reduced by a factor of $\lambda=0.95,0.7$, and 0.5 , respectively. The circle, triangle, square, and diamond dots are the GEMC simulation results. The lines are the fits to a $2 \mathrm{D}$ scaling law and the rectilinear law.

prevents us from carrying out the simulations for even lower values of $\lambda$. A typical snapshot of a vapor phase configuration is shown in Fig. 3.

As the dispersion energy decreases, the anisotropic dipolar interaction becomes more dominant in controlling the local structure of the fluid. From Fig. 3 we observe that some short chainlike structures appear. However, it seems that $\lambda=0.5$ is still too large to bring out the dipolar interaction

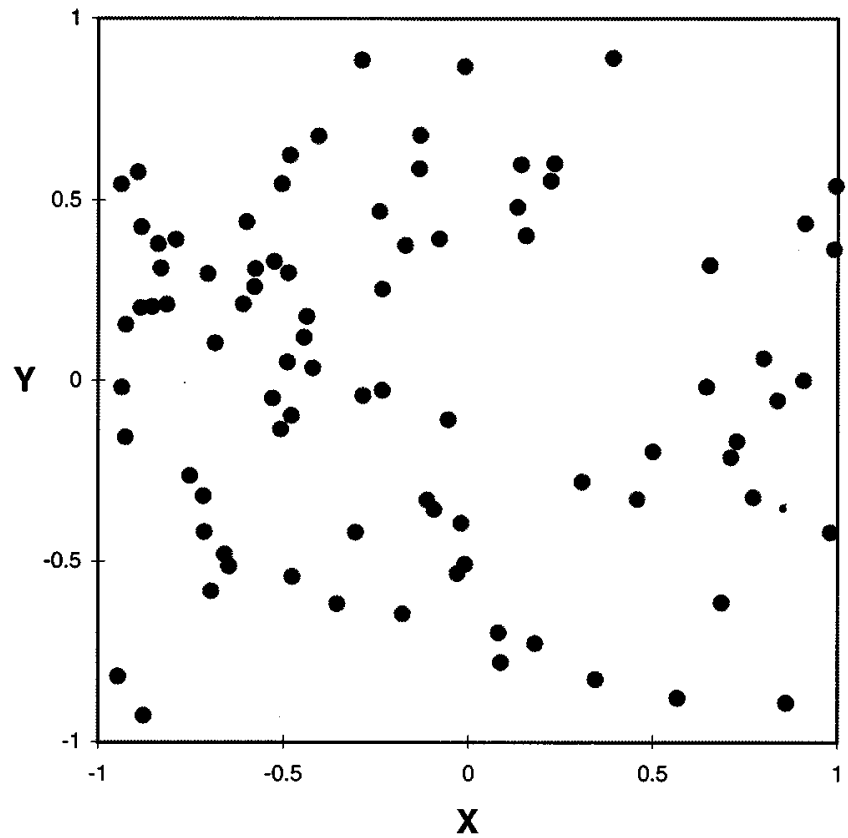

FIG. 3. A snapshot of the configuration for the quasi-2D SM fluid when $\lambda=0.5, \mu^{*}=1.0$, and $T^{*}=0.2$. 
induced short-range correlation such that all the molecules join together forming a long chain of head to tail structure and resulting in the disappearance of the critical point. Thus whether or not the critical point disappears as the dispersion energy is reduced in the quasi-2D SM system is still an open question. However, analyzing the ensemble average of $\mu_{z}^{2}$ provides us with some qualitative information. In the simulations, we found that $\left\langle\mu_{z}^{2}\right\rangle$ is always around 0.3 when $\lambda$ is 1 , but drops to 0.14 in vapor and 0.09 in liquid phases when $\lambda$ is 0.5 (at $T^{*}=0.2$ ). This indicates that the anisotropic dipolar interaction brings on a large in-plane component of dipole moment for the quasi-2D system. Therefore we speculate that when the value of $\lambda$ is sufficiently small, the molecular dipole moments could be confined in the 2D plane and thus the formation of the head to tail chainlike structures in a 2D plane is still conceivable for the quasi-2D SM system.

\section{CONCLUSION}

We have studied a quasi-2D SM system which entails essential characteristics of the system such as dipolar molecules physisorbed on a solid surface, or a Langmuir monolayer consisting of short-chain molecules with a dipolar tail. An Ewald sum germane to the quasi-2D system is, for the first time, formulated and used in the simulation to account for the long-range dipolar interaction. The vapor-liquid coexistence of the system is obtained by using the GEMC technique.

The results of the computer simulation show that the critical temperature of the quasi-2D SM system is sensitive to the strength of dipole moment whereas the critical density is not. Unlike the 3D counterpart, disappearance of vaporliquid coexistence due to reducing the dispersion energy in the potential model is not found within the practical scope of the GEMC simulation. What we found, however, is that the reduction of dispersion energy leads to a larger in-plane dipole moment. These results reflect distinctive characteristics of the dipolar system when it is confined on a 2D plane.

\section{ACKNOWLEDGMENTS}

We thank J. B. Woller and M. Gibson for their assistance in completing the manuscript and drawing the figures. This work was supported in part by the National Science Foundation and by the Office of Naval Research (XCZ). Acknowledgment is also made to the donors of the Petroleum Research Fund administered by the American Chemical Society for partial support of this work. W.W. also thanks the support by China NSF.

\section{APPENDIX A: EWALD SUM FOR THE DIPOLAR INTERACTION ENERGY IN A TWO DIMENSIONAL PERIODIC LATTICE}

Here, we will derive the formulas for the Ewald sum,

$$
V=\frac{1}{2} \sum_{i=1}^{N} \sum_{j=1}^{N} \sum_{|\mathbf{n}|=0}^{\infty}{ }^{\prime} \frac{q_{i} q_{j}}{\left|\mathbf{r}_{i j}+\mathbf{n}\right|^{2 m}}(m=1 / 2,3 / 2),
$$

where $\mathbf{n}=L\left(n_{x} \hat{i}+n_{y} \hat{j}\right), n_{x}, n_{y}=0, \pm 1, \pm 2, \ldots . L$ is the length of the simulation box, and $q$ will be defined later. The prime in Eq. (A1) means the $i=j$ term for $\mathbf{n}=0$ is excluded.

The starting point is to choose a suitable function, $\omega_{m}(\kappa r)$, to factorize $1 / r^{2 m}$, i.e.,

$$
\frac{1}{r^{2 m}}=\frac{1-\omega_{m}(\kappa r)}{r^{2 m}}+\frac{\omega_{m}(\kappa r)}{r^{2 m}},
$$

where $\omega_{m}(\kappa r)$ goes rapidly to one at large $r$ and to zero at $r=0$. Thus the first part in the right-hand side of Eq. (A2) is a short-range term and the second part is still a long-range term. Following Eq. (A2), Eq. (A1) can be factorized as

$$
V=\frac{1}{2} \sum_{i=1}^{N} \sum_{j=1}^{N} \sum_{|\mathbf{n}|=0}^{\infty} \frac{q_{i} q_{j}\left[1-\omega_{m}\left(\kappa\left|\mathbf{r}_{i j}+\mathbf{n}\right|\right)\right]}{\left|\mathbf{r}_{i j}+\mathbf{n}\right|^{2 m}}+V_{l},
$$

where $V_{l}$ is a long-range term which is written as

$$
\begin{aligned}
V_{l} & =\frac{1}{2} \sum_{i=1}^{N} \sum_{j=1}^{N} \sum_{|\mathbf{n}|=0}^{\infty} \frac{q_{i} q_{j} \omega_{m}\left(\kappa\left|\mathbf{r}_{i j}+\mathbf{n}\right|\right)}{\left|\mathbf{r}_{i j}+\mathbf{n}\right|^{2 m}} \\
& =\frac{1}{2} \sum_{i=1}^{N} \sum_{j=1}^{N} \sum_{|\mathbf{n}|=0}^{\infty} \frac{q_{i} q_{j} \omega_{m}\left(\kappa\left|\mathbf{r}_{i j}+\mathbf{n}\right|\right)}{\left|\mathbf{r}_{i j}+\mathbf{n}\right|^{2 m}}-V_{s},
\end{aligned}
$$

where in the right-hand side of Eq. (A4), the $i=j$ term for $\mathbf{n}=0$ is included in the first term. The second term,

$$
V_{s}=\frac{1}{2} \sum_{i=1}^{N} q_{i}^{2} \lim _{r \rightarrow 0} \frac{\omega_{m}(\kappa r)}{r^{2 m}}
$$

is the self-term and can be further simplified using the L'Hopital's rule.

Next, by using two relations,

$$
\sum_{\mathbf{n}} \frac{\omega_{m}\left(\kappa\left|\mathbf{r}_{i j}+\mathbf{n}\right|\right)}{\left|\mathbf{r}_{i j}+\mathbf{n}\right|^{2 m}}=\int \sum_{\mathbf{n}} \frac{\omega_{m}(\kappa r)}{r^{2 m}} \delta\left(\mathbf{r}-\mathbf{r}_{i j}-\mathbf{n}\right) d \mathbf{r}
$$

and

$$
\sum_{\mathbf{n}} \delta\left(\mathbf{r}-\mathbf{r}_{i j}-\mathbf{n}\right)=\frac{1}{A} \sum_{\mathbf{G}} \exp \left[i \mathbf{G} \cdot\left(\mathbf{r}-\mathbf{r}_{i j}\right)\right],
$$

where $\mathbf{G}=(2 \pi / L)\left(n_{x} \hat{i}+n_{y} \hat{j}\right)$ and $A\left(=L^{2}\right)$ is the area of the box, one can derive the relation

$$
\begin{aligned}
\sum_{\mathbf{n}} \frac{\omega_{m}\left(\kappa\left|\mathbf{r}_{i j}+\mathbf{n}\right|\right)}{\left|\mathbf{r}_{i j}+\mathbf{n}\right|^{2 m}}= & \frac{1}{A} \sum_{\mathbf{G}} \int \frac{\omega_{m}(\kappa r)}{r^{2 m}} \exp (i \mathbf{G} \cdot \mathbf{r}) d \mathbf{r} \\
& \times \exp \left(-i \mathbf{G} \cdot \mathbf{r}_{i j}\right) .
\end{aligned}
$$

For the quasi-2D system, a suitable choice for the function $\omega_{m}(\kappa r)$ is

$$
\omega_{m}(\kappa r)=\gamma\left(m, \kappa^{2} r^{2}\right) / \Gamma(m),
$$

where $\gamma\left(m, \kappa^{2} r^{2}\right)$ and $\Gamma(m)$ and are the complementary incomplete gamma function and the gamma function, respectively.

With Eq. (A9), the one can then evaluate analytically the two-dimensional Fourier transformation of $\gamma\left(m, \kappa^{2} r^{2}\right) / r^{2 m}$ in Eq. (A8) (see Appendix B) and $V_{s}$ in Eq. (A5); together they give the final expression for $V_{l}$. 
If $q_{i}$ and $q_{j}$ represent the charges in the system (assuming the total charge is zero, i.e., $\Sigma q_{i}=0$ ), and $m=1 / 2$, then we obtain

$$
\begin{aligned}
V_{l}= & \frac{\pi}{A} \sum_{i=1}^{N} \sum_{j=1}^{N} \sum_{\mathbf{G} \neq 0} \frac{q_{i} q_{j} \operatorname{erfc}\left(\frac{G}{2 \kappa}\right)}{G} \exp \left(-i \mathbf{G} \cdot \mathbf{r}_{i j}\right) \\
& -\sum_{i=1}^{N} \frac{\kappa q_{i}^{2}}{\sqrt{\pi}} .
\end{aligned}
$$

If $q_{i}$ and $q_{j}$ represent $z$-component of dipole moment, $\mu_{i z}$ and $\mu_{j z}$, respectively, and $m=3 / 2$, then we obtain

$$
\begin{aligned}
V_{l}= & \frac{\pi}{A} \sum_{i=1}^{N} \sum_{j=1}^{N} \sum_{\mathbf{G} \neq 0} \mu_{i z} \mu_{j z}\left[\frac{2 \kappa}{\sqrt{\pi}} \exp \left(-\frac{G^{2}}{4 \kappa^{2}}\right)\right. \\
& \left.-G \operatorname{erfc}\left(\frac{G}{2 \kappa}\right)\right] \exp \left(-i \mathbf{G} \cdot \mathbf{r}_{i j}\right) \\
& +\frac{2 \sqrt{\pi} \kappa}{A} \sum_{i=1}^{N} \sum_{i=1}^{N} \mu_{i z} \mu_{j z}-\frac{2 \kappa^{3}}{3 \sqrt{\pi}} \sum_{i=1}^{N} \mu_{i z}^{2},
\end{aligned}
$$

where $\operatorname{erfc}(x)$ is the complementary error function. Note that in deriving Eqs. (A10) and (A11), we have used the following two relations:

$$
\Gamma\left(\frac{1}{2}, x\right)=\sqrt{\pi} \operatorname{erfc}(\sqrt{x})
$$

and

$$
\Gamma(n+1, x)=n \Gamma(n, x)+x^{n} \exp (-x) .
$$

Finally replacing $q_{i}$ by $\boldsymbol{\mu}_{i s} \cdot \boldsymbol{\nabla}_{i}$ for $m=1 / 2$, the Ewald sum for the first part of Eq. (3) is

$$
\begin{aligned}
V_{\|}= & \frac{1}{2} \sum_{i=1}^{N} \sum_{j=1}^{N} \sum_{|\mathbf{n}|=0}^{\infty}{ }^{\prime}\left(\boldsymbol{\mu}_{i s} \cdot \boldsymbol{\mu}_{j s} B\left(\left|\mathbf{r}_{i j}+\mathbf{n}\right|\right)-\left(\boldsymbol{\mu}_{i s} \cdot \mathbf{r}_{i j}\right)\right. \\
& \left.\times\left(\boldsymbol{\mu}_{j s} \cdot \mathbf{r}_{i j}\right) C\left(\left|\mathbf{r}_{i j}+\mathbf{n}\right|\right)\right) \\
& +\frac{\pi}{A} \sum_{i=1}^{N} \sum_{j=1}^{N} \sum_{\mathbf{G} \neq 0} \frac{\left(\boldsymbol{\mu}_{i s} \cdot \mathbf{G}\right)\left(\boldsymbol{\mu}_{j s} \cdot \mathbf{G}\right) \operatorname{erfc}\left(\frac{G}{2 \kappa}\right)}{G} \\
& \times \exp \left(-i \mathbf{G} \cdot \mathbf{r}_{i j}\right)-\sum_{i=1}^{N} \frac{2 \kappa^{3} \mu_{i s}^{2}}{3 \sqrt{\pi}},
\end{aligned}
$$

where

$$
B(r)=\frac{\operatorname{erfc}(\kappa r)}{r^{3}}+\frac{2 \kappa}{\sqrt{\pi}} \frac{\exp \left(-\kappa^{2} r^{2}\right)}{r^{2}}
$$

and

$$
C(r)=\frac{3 \operatorname{erfc}(\kappa r)}{r^{5}}+\frac{2 \kappa}{\sqrt{\pi}}\left(\frac{3}{r^{2}}+2 \kappa^{2}\right) \frac{\exp \left(-\kappa^{2} r^{2}\right)}{r^{2}}
$$

and replacing $q_{i}$ by $\mu_{i z}$ for $m=3 / 2$ the Ewald sum for the second part of Eq. (3) is

$$
\begin{aligned}
V_{z}= & \frac{1}{2} \sum_{i=1}^{N} \sum_{j=1}^{N} \sum_{|\mathbf{n}|=0}^{\infty}, \frac{\mu_{i z} \mu_{j z}}{\left|\mathbf{r}_{i j}+\mathbf{n}\right|^{3}}\left[\operatorname{erfc}\left(\kappa\left|\mathbf{r}_{i j}+\mathbf{n}\right|\right)\right. \\
& \left.+\frac{2 \kappa\left|\mathbf{r}_{i j}+\mathbf{n}\right|}{\sqrt{\pi}} \exp \left(-\kappa^{2}\left|\mathbf{r}_{i j}+\mathbf{n}\right|^{2}\right)\right] \\
& +\frac{\pi}{A} \sum_{i=1}^{N} \sum_{j=1}^{N} \sum_{\mathbf{G} \neq 0} \mu_{i z} \mu_{j z}\left[\frac{2 \kappa}{\sqrt{\pi}} \exp \left(-\frac{G^{2}}{4 \kappa^{2}}\right)\right. \\
& \left.-G \operatorname{erfc}\left(\frac{G}{2 \kappa}\right)\right] \exp \left(-i \mathbf{G} \cdot \mathbf{r}_{i j}\right) \\
& +\frac{2 \sqrt{\pi} \kappa}{A} \sum_{i=1}^{N} \sum_{i=1}^{N} \mu_{i z} \mu_{j z}-\frac{2 \kappa^{3}}{3 \sqrt{\pi}} \sum_{i=1}^{N} \mu_{i z}^{2} .
\end{aligned}
$$

\section{APPENDIX B}

Here we show the 2D Fourier transformation

$$
\int \frac{\gamma\left(m, \kappa^{2} r^{2}\right)}{r^{2 m}} e^{i \mathbf{G} \cdot \mathbf{r}} d \mathbf{r}=\pi\left(\frac{G^{2}}{4}\right)^{m-1} \Gamma\left(-m+1, \frac{G^{2}}{4 \kappa^{2}}\right),
$$

where $\gamma(m, x)=\int_{0}^{x} e^{-t} t^{m-1} d t$ and $\Gamma(m, x)=\int_{x}^{\infty} e^{-t} t^{m-1} d t$ are the incomplete and the complementary incomplete gamma function, respectively. The equation (B1) can be rewritten as

$$
\begin{gathered}
\int_{0}^{\infty} \int_{-\pi}^{\pi} \frac{\gamma\left(m, \kappa^{2} r^{2}\right)}{r^{2 m}} e^{i G r \cos \theta} r d \theta d r \\
=\pi\left(\frac{G^{2}}{4}\right)^{m-1} \Gamma\left(-m+1, \frac{G^{2}}{4 \kappa^{2}}\right),
\end{gathered}
$$

where the inner integral of the left-hand side can be expressed in terms of the zero order Bessel function of the first kind, i.e., $J_{0}(G r)=(1 / 2 \pi) \int_{-\pi}^{\pi} e^{i G r \cos \theta} d \theta$. Therefore we only need to show the Hankel transformation

$2 \int_{0}^{\infty} \frac{\gamma\left(m, \kappa^{2} r^{2}\right)}{r^{2 m-1}} J_{0}(G r) d r=\left(\frac{G^{2}}{4}\right)^{m-1} \Gamma\left(-m+1, \frac{G^{2}}{4 \kappa^{2}}\right)$.

Let $u=G r$ and the left- and right-hand side of Eq. (B3) be $I_{L}$ and $I_{R}$, respectively; $I_{L}$ and $I_{R}$ can then be written as

$$
\begin{aligned}
I_{L}(G) & =2 G^{2(m-1)} \int_{0}^{\infty} \frac{\gamma\left(m, \frac{\kappa^{2} u^{2}}{G^{2}}\right)}{u^{2 m-1}} J_{0}(u) d u \\
& =G^{2(m-1)} I_{L}^{\prime},
\end{aligned}
$$

and

$$
I_{R}(G)=\frac{G^{2(m-1)}}{4^{m-1}} \Gamma\left(-m+1, \frac{G^{2}}{4 \kappa^{2}}\right)=G^{2(m-1)} I_{R}^{\prime},
$$

Our goal is then to show

$$
I_{L}^{\prime}(G)=I_{R}^{\prime}(G) .
$$


To proceed we first cite three auxiliary relations that will be used for the proof of Eq. (B6); (i) A theorem related to the incomplete gamma functions,

$$
\frac{d \gamma(m, x)}{d x}=-\frac{d \Gamma(m, x)}{d x}=x^{-m} e^{-x}
$$

(ii) the integral formula, ${ }^{31}$

$$
\int_{0}^{\infty} x e^{-\alpha x^{2}} J_{0}(x) d x=\frac{1}{2 \alpha} e^{-1 / 4 \alpha^{2}},
$$

and (iii) two limiting cases for the incomplete gamma functions, $\gamma(m, 0)=0$, and $\Gamma(m, \infty)=0$.

From Eqs. (B4), (B7), and (B8), we obtain

$$
\begin{aligned}
\frac{d I_{L}^{\prime}}{d G} & =-4 \frac{\kappa^{2 m}}{G^{2 m+1}} \int_{0}^{\infty} u e^{-\kappa^{2} u^{2} / G^{2}} J_{0}(u) d u \\
& =-4 \frac{\kappa^{2(m-2)}}{G^{2 m-1}} e^{-G^{2} / 4 \pi^{2}} .
\end{aligned}
$$

On the other hand, from Eqs. (B5) and (B7), we obtain

$$
\frac{d I_{R}^{\prime}}{d G}=-4 \frac{\kappa^{2(m-2)}}{G^{2 m-1}} e^{-G^{2} / 4 \kappa^{2}} .
$$

Thus, we have

$$
\frac{d I_{L}^{\prime}}{d G}=\frac{d I_{R}^{\prime}}{d G} .
$$

This equality of the derivative of $I_{L}^{\prime}$ and $I_{R}^{\prime}$ in conjunction with the auxiliary relation (iii) (for $\left.I_{L}^{\prime}\right|_{G=\infty}$ and $\left.I_{R}^{\prime}\right|_{G=\infty}$ ) gives rise to Eq. (B6), or, Eq. (B1).

${ }^{1}$ W. A. Steele, The Interaction of Gases with Solid Surfaces (Pergamon, Oxford, 1974).

${ }^{2}$ D. J. Shaw, Introduction to Colloid and Surface Chemistry (Butterworths, London, 1980)

${ }^{3}$ M. Elbaum and S. G. Lipson, Phys. Rev. Lett. 72, 3562 (1994).
${ }^{4}$ M. Rami Reddy and S. F. O'Shea, Can. J. Phys. 64, 677 (1986).

${ }^{5}$ M. Rovere, J. Phys. Condensed Matter 5, B193 (1993); M. Rovere, D. W. Heermann, and K. Binder, ibid. 2,7009 (1990).

${ }^{6}$ K. P. Shukla and M. Robert, Fluid Phase Equilib. 79, 139 (1992); L. J. Chen, M. Rober, and K. P. Shukla, J. Chem. Phys. 93, 8254 (1990).

${ }^{7}$ J. H. Sikkenk, J. M. J. van Leeuwen, E. O. Vossnack, and A. F. Bakker, Physica A 146, 622 (1987); J. H. Sikkenk, H. J. Hilhorst, and A. F. Bakker, Physica 131A, 587 (1985).

${ }^{8}$ L. Mederos, E. Chacon, and G. Navascues, Mol. Phys. 54, 211 (1985).

${ }^{9}$ R. R. Singh, K. S. Pitzer, J. J. de Pablo, and J. M. Prausnitz, J. Chem. Phys. 92, 5463 (1990).

${ }^{10}$ B. Smit and D. Frenkel, J. Chem. Phys. 94, 5663 (1991).

${ }^{11}$ S. Jiang and K. E. Gubbins, Mol. Phys. 86, 599 (1995).

${ }^{12}$ L. D. Landau and E. M. Liftshitz, Statistical Physics, Part 1 (Pergamon, Oxford, 1980)

${ }^{13}$ G. Bossis, Mol. Phys. 38, 2023 (1979); G. Bossis, B. Quentrec, and C. Brot, ibid. 39, 1233 (1980); G. Bossis, C. Brot, and C. Hesse-Bezot, ibid. 40, 1053 (1980).

${ }^{14}$ S. W. de Leeuw, J. W. Perram, and E. R Smith, Proc. R. Soc. London, Ser. A 373, 27, 57 (1980)

${ }^{15}$ S. W. de Leeuw, J. W. Perram, and E. R. Smith, Annu. Rev. Phys. Chem. 37, 245 (1986).

${ }^{16}$ M. P. Allen and D. J. Tildesley, Computer Simulation of Liquids (Clarendon, Oxford, 1989).

${ }^{17}$ B. R. A. Nijbore and F. W. de Wette, Physica 23, 309 (1957).

${ }^{18}$ A. Z. Panagiotopoulos, Mol. Phys. 61, 813 (1987).

${ }^{19}$ A. Z. Panagiotopoulos, N. Quirke, M. Stapleton, and D. J. Tildesley, Mol. Phys. 63, 527 (1988).

${ }^{20}$ A. Z. Panagiotopoulos, Mol. Simul. 9, 1 (1992).

${ }^{21}$ B. Smit and D. Frenkel, Mol. Phys. 68, 951 (1989).

${ }^{22}$ J. M. Yeomans, Statistical Mechanics of Phase Transitions (Clarendon, Oxford, 1991).

${ }^{23}$ J. R. Recht and A. Z. Panagiotopoulos, Mol. Phys. 80, 843 (1993).

${ }^{24}$ M. E. van Leeuwen, Mol. Phys. 82, 383 (1994).

${ }^{25}$ M. Bienfait, in Phase Transitions in Surface Films, edited by J. G. Dash and J. Ruvalds (Plenum, New York and London, 1980).

${ }^{26}$ M. A. Osipov, P. I. C. Teixeira, and M. M. Telo da Gama, Phys. Rev. E 54, 2597 (1996).

${ }^{27}$ M. E. van Leeuwen and B. Smit, Phys. Rev. Lett. 71, 3991 (1993).

${ }^{28}$ M. J. Stevens and G. S. Grest, Phys. Rev. E 51, 5962 (1995).

${ }^{29}$ J. J. Weis and D. Levesque, Phys. Rev. Lett. 71, 2729 (1993).

${ }^{30}$ D. Levesque and J. J. Weis, Phys. Rev. E 49, 5131 (1994).

${ }^{31}$ Table of Integral Series, and Products, edited by I. S. Gradshteyn and I. M. Ryzhik (Academic, Orlando, 1980), p. 717. 